\title{
Inclusive diffraction at HERA
}

\author{
Laurent Favart \\ On behalf of the HI and ZEUS Collaborations \\ Université Libre de Bruxelles, \\ CP 230, Bd. du Triomphe \\ B-1040 Brussels, Belgium \\ lfavart@ulb.ac.be
}

\begin{abstract}
Results are reported on recent measurements, performed by the H1 and ZEUS Collaborations, of the cross section of the diffractive deep-inelastic process $e p \rightarrow e X p$ using different experimental methods. In particular, first results using the Very Forward Proton Spectrometer of H1 are discussed.
\end{abstract}

Keywords: HERA, Diffraction, QCD, Structure functions, Vector mesons

PACS: PACS 13.60.-r, 13.85.-t

\section{INTRODUCTION}

The diffractive interaction is a feature of hadron-hadron scattering at high energy corresponding to a $t$-channel exchange of the vacuum quantum numbers and a small momentum transfer. It has been described, in the past, in the framework of Regge theory, where the exchange was interpreted as the Pomeron $(\mathbb{P})$ trajectory, characterized by a weak energy dependence, in particular, with respect to the fast decrease of the total cross section at smaller energy due to Reggeon $(\mathbb{R})$ exchange: $\sigma_{T o t}=B W^{2\left(\alpha_{\mathbb{R}}-1\right)}+A W^{2\left(\alpha_{P}-1\right)}$, where $W$ is the center of mass energy, $A$ and $B$ normalisation factors and typically $\alpha_{\mathbb{R}}=0.55$ and $\alpha_{\mathbb{P}}=1.08$.

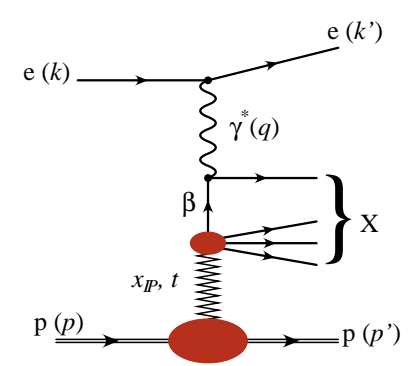

FIGURE 1. Basic diagram for diffraction in presence of a hard scale at in ep collisions.

Diffraction has two distinguishing features. First, hadron emission from the exchange itself being suppressed by its colourless nature, the two diffractively dissociated systems are separated in rapidity space, forming a large rapidity gap (LRG). Secondly, the diffractive events are observed with a small momentum transfer in both the transverse and longitudinal coordinates. The four-momentum of the exchange, $t$, and the longitudinal momentum fraction of the exchange, $x_{\mathbb{P}}$, are both small; $|t|$ is typically less than the square of the nucleon mass and $x_{\mathbb{P}}$ is smaller than 0.05 .

The high energies of the HERA ep collider allowed us for the first time to study diffraction in terms of perturbative QCD (pQCD), i.e. in the presence of a hard scale (called hard diffraction). At HERA the diffractive interaction takes place between the hadronic behaviour of the exchanged virtual photon and the proton (see Fig. 1). 
This article concentrates on different measurements performed at HERA by the H1 and ZEUS Collaborations on inclusive hard diffraction. Their analysis and interpretation in terms of pQCD is discussed in [1].

\section{Kinematics and total cross section}

The inclusive diffractive cross section at HERA, $e p \rightarrow e X p$, can be defined with the help of four kinematic variables conveniently chosen as $Q^{2}, x_{\mathbb{P}}, \beta$ and $t$. The variable $\beta$ is the fraction of the exchanged momentum carried by the quark struck by the photon, or in other terms, the fraction of the exchanged momentum reaching the photon. These variables are related to the Bjorken $x_{B j}$ scaling variable by the relation $x_{B j}=\beta \cdot x_{\mathbb{P}}$. The presence of the hard scale, $Q^{2}$, ensures that the virtual photon is point-like and that the photon probes the partonic structure of the diffractive exchange (Fig. 1), in analogy with the inclusive DIS processes. Also by analogy, the measured cross section is expressed in the form of two four-fold diffractive structure functions $F_{2}^{D(4)}$ and $F_{L}^{D(4)}$ according to

$$
\frac{\mathrm{d}^{4} \sigma(e p \rightarrow e X p)}{\mathrm{d} Q^{2} \mathrm{~d} x_{\mathbb{P}} \mathrm{d} \beta \mathrm{d} t}=\frac{4 \pi \alpha^{2}}{\beta Q^{4}}\left[Y_{+} F_{2}^{D(4)}\left(Q^{2}, x_{\mathbb{P}}, \beta, t\right)-y^{2} F_{L}^{D(4)}\left(Q^{2}, x_{\mathbb{P}}, \beta, t\right)\right]
$$

where $Y_{+}=1+(1-y)^{2}$ and $y$ is the usual Bjorken variable, $y \simeq W^{2} / s$. The reduced cross section, used later, is defined as $\sigma_{r}^{D(4)}=F_{2}^{D(4)}-y^{2} / Y_{+} F_{L}^{D(4)}$. For $y$ not too close to unity, $\sigma_{r}^{D(4)}=F_{2}^{D(4)}$ holds to very good approximation.
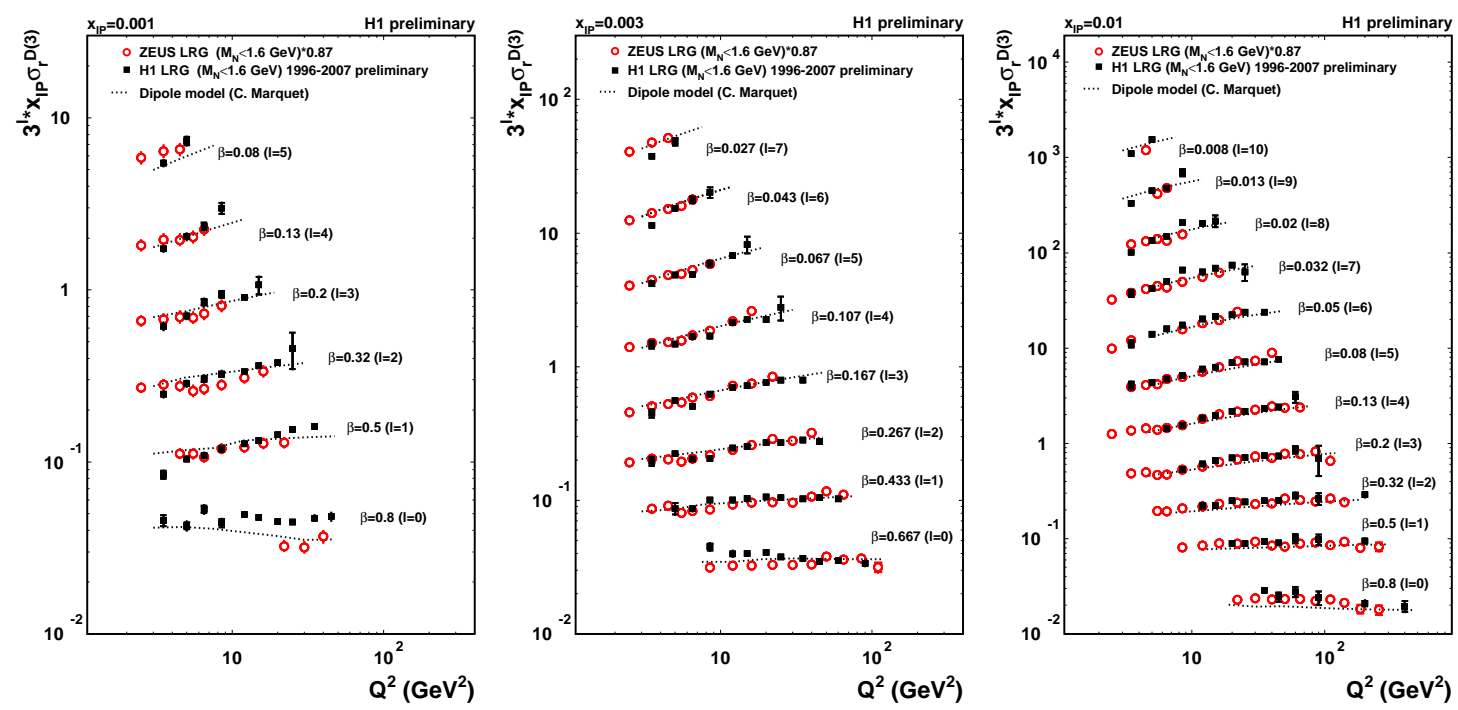

FIGURE 2. H1-prel.[7] and ZEUS[4] measurements of the diffractive reduced cross section. 


\section{CROSS SECTION MEASUREMENTS AND COMPARISONS}

Experimentally, diffractive processes are accessed either by tagging the outgoing proton in the H1 Forward Proton Spectrometer or the ZEUS Leading Proton Spectrometer ('LPS method' $[2,4,5,6]$ ) or by requiring the presence of a large gap in the rapidity distribution of hadronic final state particles in the forward region ('LRG method' [3, 4, 7]). A third approach, not considered in detail here, is based on the $M_{X}$ distribution shape [8]. Whilst the LRG and $M_{X}$-based techniques yield better statistics than the LPS method, they suffer from systematic uncertainties associated with an admixture of proton dissociation to low mass states, which is irreducible due to the limited forward detector acceptance.

The H1 Collaboration recently released preliminary measurement based on LRG technique applied to HERA-II data [7]. These results are compared to ZEUS most recent measurement [4] in Fig. 2. The normalisation differences between the two experiments of $13 \%$ is compatible with the uncertainty on p-diss. correction $(8 \%)$ and relative normalisation uncertainty (7\%). After correcting for this factor, a good agreement is observed between the shapes of the $\mathrm{H} 1$ and ZEUS cross sections throughout most of the phase space studied. The few $\%$ point-to-point precision is reached over wide kinematic range.

Performing a fit of the cross section with a form à la Regge (see introduction) one founds that the Pomeron intercept, $\alpha_{I P}$ does not depend on $Q^{2}$, in strong contrast to what is measured in inclusive DIS. This confirms the dominance of nonperturbative effects in the Pomeron. Using e.g. H1 FPS from HERA II data [6] one gets $\alpha_{I P}(0)=1.10 \pm 0.02(\mathrm{exp}) \pm 0.03$ (model) compatible with a soft pomeron.

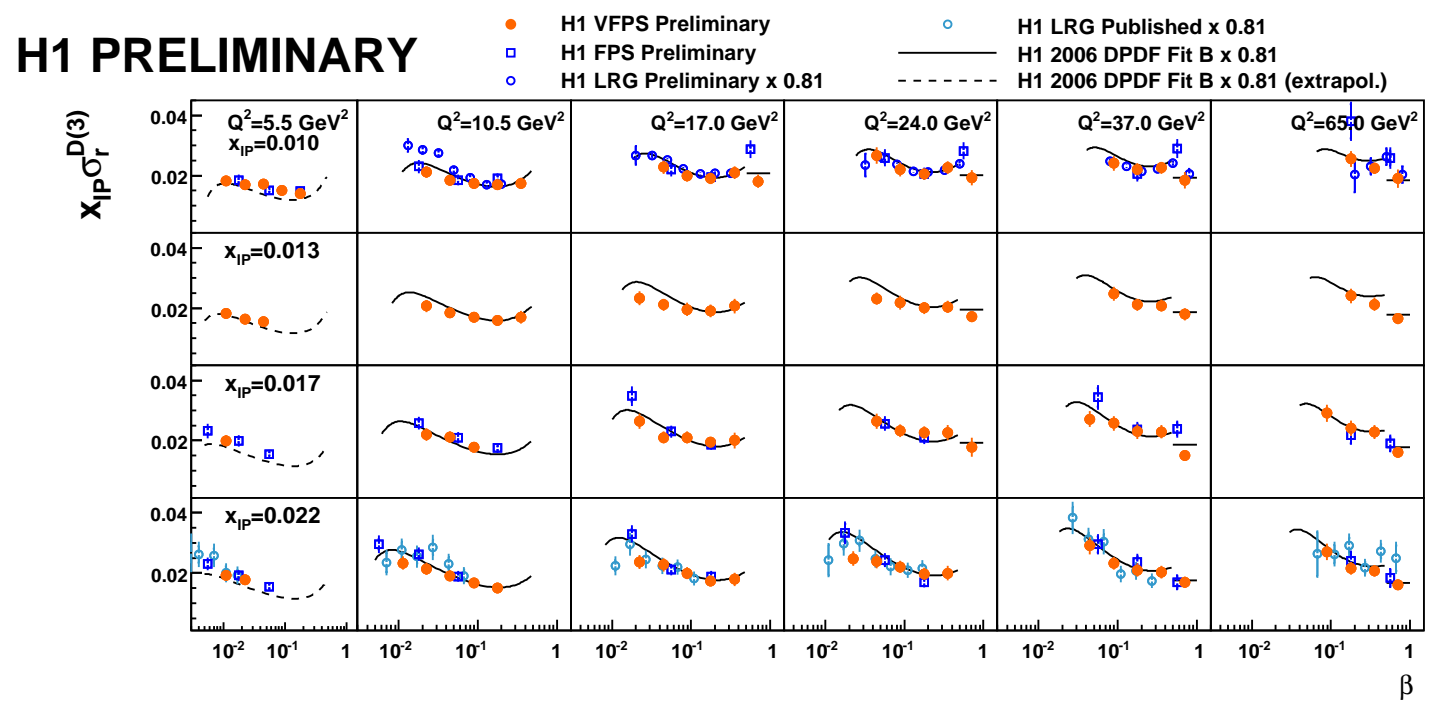

FIGURE 3. Comparison of H1-prel reduced cross section measurement using VFPS [5], FPS [6] and LRG [7] technics. 


\section{The Very Forward Proton Spectrometer of H1}

The Very Forward Proton Spectrometer (VFPS) with an acceptance above $90 \%$ in a restricted $x_{\mathbb{P}}$ range $\left(x_{\mathbb{P}} \in[0.009,0.025]\right)$ has been installed in the proton beam line of the H1 experiment for the HERA-II phase. The spectrometer, located at $220 \mathrm{~m}$ downstream of the interaction point is based on the Roman Pot technique and consists of two stations situated in the cold section of the proton beam line. The VFPS high performances (efficiency of $96 \%$, normalisation uncertainty below $5 \%, x_{\mathbb{P}}$ resolution of $12 \%$, and background contribution below 1\%) allows improved precision measurements.

The preliminary measurement of the reduced cross section performed with the VFPS is compared to preliminary H1 LRG [7] and H1 FPS [6]) in Fig. 3. A good agreement is found. The most direct test of agreement is performed by taking the ratio of VFPS over FPS and is found to be VFPS/FPS $=0.96 \pm 0.02$ (stat) \pm 0.11 (syst) \pm 0.08 (norm).

\section{The $t$ dependence}

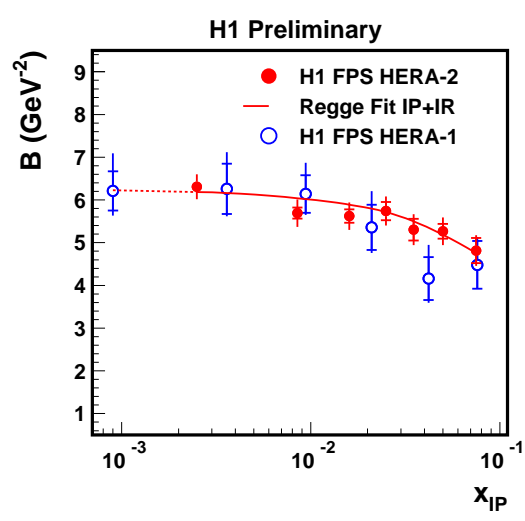

FIGURE 4. $t$ dependence of the cross section (see text)
Recent H1-FPS measurement [6] provide an improved measurement of the $t$ dependence of the cross section. An exponential of the form $\exp (-B|t|)$ is fitted to the cross section. The dependence of $b$ with $x_{I P}$ is shown in Fig. 4 and compared to previous $\mathrm{H} 1$ FPS results [2]. The $x_{\mathbb{P}}$ dependence is compatible with the Regge model in which the $t$ slope results from two contributions with different $b$ values. The parameter $B$ is found to be compatible with no $Q^{2}$ nor $\beta$ dependence. This contrast with diffractive exclusive final state production (DVCS, $\rho, \phi, \ldots$ ) where a strong dependence in $Q^{2}$ is measured (see e.g. [9]).

\section{REFERENCES}

1. A. Solano, these proceedings.

2. H1 Coll., Eur. Phys. J. C 48 (2006) 749 [hep-ex/0606003].

3. H1 Coll., Eur. Phys. J. C 48 (2006) 715 [hep-ex/0606004].

4. ZEUS Coll., Nucl. Phys. B 816 (2009) 1 [hep-ex/0812.2003].

5. H1 Coll., 'Measurement of diffractive DIS with with the VFPS' [H1prelim-10-014].

6. H1 Coll., 'Measurement of diffractive DIS with a leading proton at HERA-2' [H1prelim-09-012] and [H1prelim-10-012].

7. H1 Coll., 'Measurement of Inclusive Diffractive DIS at HERA (99-04 data)' [H1prelim-10-011].

8. ZEUS Coll., Nucl. Phys. B 800 (2008) 1 [hep-ex/0802.3017].

9. L. Favart, "Vector Mesons at HERA", proceedings of Diffraction 2008. 